\title{
Influence of Institution-Based Factors on Preoperative Blood Testing Prior to Low-Risk Surgery: A Bayesian Generalized Linear Mixed Approach
}

\author{
Kazuki Ide, ${ }^{1,2}$ Hiroshi Yonekura, ${ }^{1}$ Yohei Kawasaki, ${ }^{1,3}$ and Koji Kawakami ${ }^{1,2}$ \\ ${ }^{1}$ Department of Pharmacoepidemiology, Graduate School of Medicine and Public Health, Kyoto University, Yoshida-konoecho, \\ Sakyo-ku, Kyoto 606-8501, Japan \\ ${ }^{2}$ Center for the Promotion of Interdisciplinary Education and Research, Kyoto University, Yoshida-honmachi, Sakyo-ku, \\ Kyoto 606-8501, Japan \\ ${ }^{3}$ Clinical Research Center, Chiba University Hospital, 1-8-1 Inohana, Chuo-ku, Chiba 260-8677, Japan \\ Correspondence should be addressed to Koji Kawakami; kawakami.koji.4e@kyoto-u.ac.jp
}

Received 23 March 2017; Revised 7 October 2017; Accepted 13 November 2017; Published 7 December 2017

Academic Editor: Chuangyin Dang

Copyright (C) 2017 Kazuki Ide et al. This is an open access article distributed under the Creative Commons Attribution License, which permits unrestricted use, distribution, and reproduction in any medium, provided the original work is properly cited.

To optimize delivery of health care services in clinical practice, the use of unnecessary interventions should be reduced. Although recommendations for this reduction have been accepted worldwide, recent studies have revealed that the use of such procedures continues to increase. We conducted a retrospective cohort study using a nationwide claim-based database to evaluate factors influencing preoperative blood testing prior to low-risk surgery, via a Bayesian generalized linear mixed approach. The study period was set from April 1, 2012, to March 31, 2016, and 69,252 surgeries performed at 9,922 institutions were included in the analysis. Mean patient age was $44.3 \pm 11.3$ years ( $57 \%$ female). Preoperative blood tests were performed for $59.0 \%$ of procedures. Among institutional factors, the number of beds was strongly associated with preoperative blood testing (odds ratio [95\% highest posterior density interval (HPD interval)], 2.64 [2.53 to 2.75]). The difference (95\% credible interval) in the rate of preoperative blood testing between institutions with $<100$ beds and $\geq 100$ beds was 0.315 [ 0.309 to 0.322 ], and the Bayesian index $\theta$ was 1.00 . This indicated that preoperative blood tests are strongly influenced by institutional factors, suggesting that specific guidelines should be developed to avoid excessive preoperative testing for low-risk surgery.

\section{Introduction}

To optimize the delivery of health care services in clinical practice, the use of unnecessary and/or non-evidence-based tests, treatments, and procedures should be reduced $[1,2]$. In addition to incurring unnecessary costs, previous studies have revealed that routine preoperative tests neither decrease adverse events nor improve surgical outcomes [3-6]. In 2012, the American Board of Internal Medicine Foundation (ABIMF) launched the Choosing Wisely campaign, which encourages physicians and patients to communicate regarding the most appropriate tests and treatments for each patient $[1,7]$. Since then, various countries including Japan have adopted similar campaigns $[7,8]$, and many specialty societies have recommended that routine preoperative testing be avoided for patients undergoing low-risk surgery [9-11]. The National Institute for Health and Care Excellence (NICE) in the UK also discourages excessive preoperative testing unless such testing yields clinical information that cannot be obtained from a patient's history and physical examination [12].

Although these campaigns and recommendations have been accepted worldwide, recent studies have revealed that the use of unnecessary tests continues to increase [13]. Previous studies have conceptualized the problem using worldwide system-level metrics to examine factors influencing the realworld situation in each country. Therefore, we conducted a retrospective cohort study using a nationwide claim-based database to evaluate factors influencing the use of preoperative blood tests prior to low-risk surgery. Specifically, we 
evaluated the fixed effects of the Bayesian generalized linear mixed model using Markov Chain Monte Carlo methods when examining the influence of institutional factors [14]. We also performed Bayesian conjugate analysis to compare the rate of preoperative blood testing among groups, allowing us to calculate the Bayesian index $\theta$, which can be used to determine the probability that the hypothesis is true and the credible intervals (CrIs) contain the true parameter [15].

\section{Materials and Methods}

2.1. Study Overview. We conducted a retrospective cohort study using an insurance claim-based database covering approximately five million insured individuals in Japan since 2005. Individuals who had undergone low-risk surgery were included in the analysis. Factors influencing the use of preoperative blood tests were explored, following which institutions were divided into two groups based on the number of beds. The differences between the two groups and the probability of hypothesis truth were then evaluated.

2.2. Study Population. The insurance claim-based database was provided by the Japan Medical Data Center Co., Ltd. (JMDC; Tokyo, Japan), and the study period was set from April 1, 2012, to March 31, 2016. The database included the following information: sex, age, medical and pharmacy claim data (outpatient as well as inpatient data), clinical diagnostic codes (International Classification of Disease 10th revision [ICD-10]), drug prescription information codes (World Health Organization Anatomical Therapeutic Chemical classification codes [ATC codes]), and standardized procedure codes (Japan-specific standardized procedure codes [K codes]).

Low-risk surgery was defined according to $\mathrm{K}$ codes for ophthalmologic, superficial, breast, thyroid, minor gynecological, orthopedic [arthroscopy], and minor urological procedures, based on the findings of previous studies [9, 16]. Patients aged 20-64 years with at least 12 months of insurance data prior to undergoing low-risk surgery during the study period were included. Individuals without any required clinical data and those who had undergone lowrisk procedures in an inpatient setting in conjunction with additional/emergency procedures were excluded.

2.3. Outcomes. The primary outcome measure of the present study was the presence or absence of preoperative blood tests prior to low-risk surgery. We used a Bayesian generalized linear mixed approach to estimate the coefficients of each variable (patient variables and institutional factors) for preoperative blood tests. Preoperative tests were defined as those ordered within 60 days of the index procedure [17] and included the following: complete blood count (CBC), basic metabolic panel, coagulation tests, and liver function tests (LFTs). In Japan, health insurance coverage of these preoperative tests does not differ based on the patient's health insurance status. Institutional differences in preoperative testing rates were regarded as the secondary outcome measure.
2.4. Statistical Analysis. Continuous variables are summarized as mean and standard deviations (SD), while categorical variables are summarized as frequencies and proportions (\%).

In the present study, we evaluated differences in preoperative testing among institutions using a Bayesian generalized linear mixed model $[14,18]$. Institutions were regarded as random effects, and the model contained two levels of hierarchical structure.

In this model, $Y_{i j}$ indicates whether the $j$ th individual in the $i$ th institution had undergone preoperative blood testing. Thus, $Y_{i j}$ represents the Bernoulli distribution of parameter $\pi_{i}$. The rate $\pi_{i}$ represents the rate of preoperative blood testing at each institution. We assumed that the random effect $c$ had a mean of 0 and a normal distribution for variance $\sigma_{0}^{2}$. The hyperparameter $\sigma_{0}^{2}$ was assumed to follow a gamma distribution, which does not take negative values, as it represents the variance. The shape and scale parameters of the gamma distribution were each set to 0.001, assuming a fixed effect with a mean of 0 and a normally distributed variance of 10,000. Using these parameters, the model can be expressed as follows:

$$
\begin{aligned}
& \log \left(\frac{\pi_{i}}{1-\pi_{i}}\right)= \beta_{0}+\beta_{1} x_{1 i j}+\beta_{2} x_{2 i j}+\cdots+\beta_{k} x_{k i j}+\cdots \\
&+\beta_{p} x_{p i j}+c \\
& \beta_{k i j} \sim N(0,1000), \\
& c \sim N\left(0, \sigma_{0}^{2}\right), \\
& \sigma_{0}^{2} \sim \text { Gamma }(0.001,0.001),
\end{aligned}
$$

where $\beta_{k}$ represents the fixed-effect parameter and $x_{k i j}$ represents the $j$ th individual in the $i$ th institution. All patient variables (age [reference: $<25$ years], sex [reference: male], Charlson comorbidity index [CCI; reference: 0-1], antiplatelet use [reference: nonuse], anticoagulant use [reference: nonuse], angiotensin-converting enzyme inhibitor/angiotensin-receptor blocker use [reference: nonuse], diuretic use [reference: nonuse], chemotherapy [reference: nonchemotherapy], type of anesthesia [reference: local anesthesia], ophthalmologic procedure [reference: nonophthalmologic procedure], and surgical setting [reference: inpatient setting]) and institutional factors (number of beds [reference: <100], hospital status [reference: nonteaching hospital], and number of operations [reference: Q1 \{lowest\}]) were included as predictors in the Bayesian generalized linear mixed model. We used the aforementioned expression to calculate the posterior mean and the standard error of each parameter as well as the $95 \%$ highest posterior density (95\% HPD) interval. We calculated the posterior distribution using Markov Chain Monte Carlo methods by applying the Metropolis-Hastings method to the calculation algorithm. A total of 20,000 samples were calculated, and the first 500 samples were discarded as burn-in. We used graphical plots to interpret the convergence of the MCMC results [19]. The posterior mean of each parameter was used as the model coefficient, and these values were expressed as odds ratios $\left(\exp \left[\beta_{k}\right]=\right.$ odds ratio $)$. 
We calculated the number of preoperative blood tests and compared the rate of testing between institutions with $<100$ beds and $\geq 100$ beds. We calculated the $95 \%$ credible interval (CrI) of the difference in the rate of preoperative blood testing using an exact method. We then compared rates of preoperative blood testing between the two institution groups using the Bayesian index proposed by Kawasaki and Miyaoka [15]. The threshold of 100 beds has previously been used as an institutional criterion for categorizing general hospitals in Japan, and a recent survey in Japan has also demonstrated the medical relevance of this threshold [20]. Therefore, we chose to use a threshold of 100 beds.

In the present study, we set $z_{1}$ as the number of preoperative blood tests at institutions with $<100$ beds and $z_{2}$ as that for institutions with $\geq 100$ beds. We regarded $Z_{1}$ and $Z_{2}$ as binomial random variables for $n_{1}$ preoperative blood tests and $n_{2}$ preoperative blood tests and parameters $p_{1}$ and $p_{2}$, respectively. The conjugate prior density for $p_{1}$ is the beta distribution with parameters $\alpha_{i}$ and $\beta_{i}$, with parameters $\alpha_{i}>$ $0, \beta_{i}>0$, and $i=1,2$. The prior distribution was regarded as noninformative and defined as beta $(1,1)$. The posterior pdf for $p_{1}$ is the proposed beta distribution with parameters $a_{i}=\alpha_{i}+z_{i}$ and $b_{i}=n_{i}-\alpha_{i}+\beta_{i}$. Using the posterior pdf, we calculated the exact Bayesian index $\theta$. The accurate expression of probability for Bayesian index $\theta$ is proposed as follows:

$$
\begin{gathered}
P\left(p_{1}<p_{2} \mid z_{1}, z_{2}\right)=\frac{B\left(a_{1}+a_{2}, b_{1}\right)}{a_{2} B\left(a_{1}, b_{1}\right) B\left(a_{2}, b_{2}\right)} \\
\cdot{ }_{3} F_{2}\left(\begin{array}{c}
a_{2}, 1-b_{2}, a_{1}+a_{2} \\
1+a_{2}, a_{1}+a_{2}+b_{1}
\end{array} ; 1\right),
\end{gathered}
$$

where ${ }_{3} F_{2}\left(\begin{array}{c}k_{1}, k_{2}, k_{3} \\ l_{1}, l_{2}\end{array} 1\right)=\sum_{t=0}^{\infty}\left(\left(k_{1}\right)_{t}\left(k_{2}\right)_{t}\left(k_{3}\right)_{t} /\left(l_{1}\right)_{t}\left(l_{2}\right)_{t}\right)(1 / t !)$, $k_{1}+k_{2}+k_{3}<l_{1}+l_{2}$, represent hypergeometric series and $(k)_{i}$ is the Pochhammer symbol.

All statistical analyses were performed using SAS version 9.4 for Windows (SAS Institute Inc., Cary, NC, USA). When performing the analysis using the Bayesian generalized linear mixed model, we used the MCMC procedure of SAS. A general SAS code for this analysis is included in Appendix 2.

2.5. Ethical Considerations. The present study was approved by the Ethics Committee of Kyoto University Graduate School and Faculty of Medicine (number R0800, September $8,2016)$. This study was conducted in accordance with the Declaration of Helsinki and the Ethical Guidelines for Medical and Health Research Involving Human Subjects in Japan [21, 22]. Additional informed consent was not required based on these guidelines. The Strengthening the Reporting of Observational Studies in Epidemiology (STROBE) criteria were applied in the reporting of the present study [23].

\section{Results}

3.1. Clinical Characteristics of Participants. The flow diagram for the present study is shown in Figure 1, and patient characteristics are presented in Table 1.

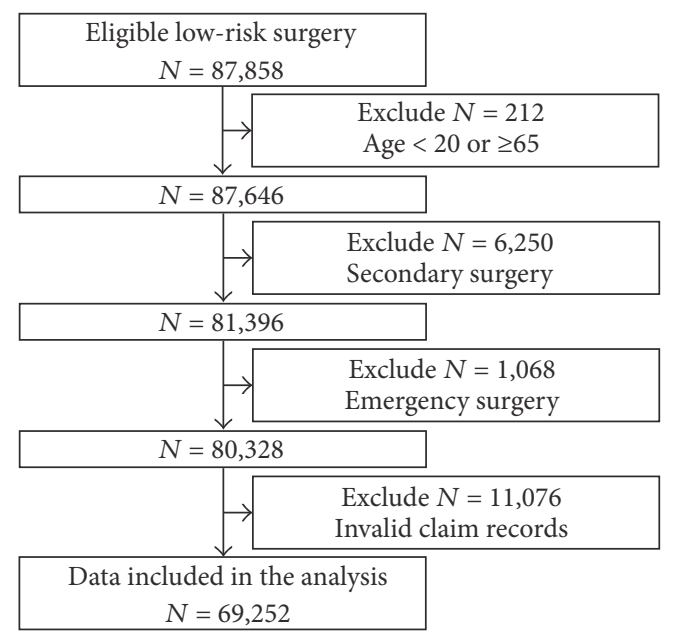

Figure 1: Study flow diagram.

During the study period, 3,543,575 individuals from 65,371 institutions were included in the JMDC database. Among them, 70,244 individuals had undergone a total of 87,858 low-risk surgical procedures. Following the exclusion of 18,606 cases that met exclusion criteria, 69,252 surgeries performed at 9,922 institutions were included in the final analysis. Mean age (SD) in this cohort was 44.3 years (11.3 years), and $57.0 \%$ of patients were female. Local anesthesia was most frequently performed. Inpatient procedures accounted for $31.0 \%$ of surgical cases, and $40.6 \%$ of institutions had $\geq 100$ beds.

3.2. Rate of Preoperative Blood Testing and Bayesian Generalized Linear Mixed Approach. Preoperative blood tests were performed for $59.0 \%$ of procedures. The prevalence of each preoperative blood test was as follows: CBC, 57.8\%; basic metabolic panel, $49.6 \%$; LFTs, $48.0 \%$; coagulation test, $35.6 \%$. The odds ratio of each variable (patient variables and institutional factors) with 95\% HPD intervals for preoperative blood testing is presented in Table 2. Among patient variables, general anesthesia (5.42 [4.85 to 6.03]), anticoagulant use (3.57 [2.22 to 5.61]), and regional anesthesia (3.14 [2.89 to 3.44]) were relatively associated with preoperative blood testing. Among institutional factors, the number of beds within an institution was strongly associated with preoperative blood testing (odds ratio [95\% HPD interval], 2.64 [2.53 to 2.75]). The graphical plots used to interpret the convergence of the MCMC results are included in Appendix 1.

3.3. Differences in Preoperative Blood Testing and Bayesian Index. The posterior beta distribution parameters were $a_{1}=$ $21829+1=21830$ and $b_{1}=28095-21829+1=6267$ for institutions with $\geq 100$ beds and $a_{2}=18993+1=18994$ and $b_{2}=41157-18993+1=22165$ for institutions with $<100$ beds. The difference $[95 \% \mathrm{CrI}]$ in the rate of preoperative blood testing between institutions with $<100$ beds and $\geq 100$ beds was 0.315 [ 0.309 to 0.322 ], and the Bayesian index $\theta$ was 1.00 (Table 3 ). These findings indicate a point estimate of a $31.5 \%$ difference in preoperative blood testing, with a true 
TABle 1: Patient characteristics.

\begin{tabular}{lc}
\hline Characteristics & \\
\hline$N$ & 69,252 \\
Age, yr & \\
$\quad<25$ & $3,070(4.4)$ \\
$25-34$ & $13,172(19.0)$ \\
$35-44$ & $19,816(28.6)$ \\
$45-54$ & $18,281(26.4)$ \\
$55-64$ & $14,913(21.5)$ \\
Sex, female & $39,489(57.0)$ \\
CCI score & \\
$0-1$ & $57,106(82.5)$ \\
2 & $6,742(9.7)$ \\
M3 & $5,404(7.8)$ \\
Medication & \\
Antiplatelet & $1,759(2.5)$ \\
Anticoagulant & $330(0.5)$ \\
ACEI/ARB & $5,399(7.8)$ \\
Diuretics & $1,075(1.6)$ \\
Chemotherapy & $792(1.1)$ \\
Type of anesthesia & \\
General anesthesia & \\
Regional anesthesia & \\
Sedation & \\
Local anesthesia & $28,095(40.6)$ \\
Unknown & \\
Inpatient & \\
Outpo & \\
Number of beds & \\
\hline
\end{tabular}

ACEI, angiotensin-converting enzyme inhibitor; ARB, angiotensin-receptor blocker; CCI, Charlson comorbidity index.

difference between $30.9 \%$ and $32.2 \%$. Based on the value of the Bayesian index $\theta$, this difference can be observed as $100 \%$.

\section{Discussion}

The present study aimed to evaluate factors influencing preoperative blood testing prior to low-risk surgery among individuals in a nationwide claim-based database using Bayesian approaches. Our results indicated that the rate of preoperative blood testing is strongly influenced by institutional factors, such as institution size. Furthermore, our results suggested that patient factors were also associated with preoperative blood tests. However, the influence of institutional factors remained after adjusting for these variables, indicating that modification of practices at the institutional level is necessary to reduce unnecessary preoperative blood testing.

In this study, we utilized a nationwide claim-based database covering 4.7 million insured individuals treated at
TABLE 2: Odds ratio for patient and institutional factors.

\begin{tabular}{|c|c|}
\hline Variables & OR [95\% HPD interval] \\
\hline \multicolumn{2}{|l|}{ Patient factors } \\
\hline Age & $1.09[1.07$ to 1.11$]$ \\
\hline Sex (female) & $1.03[0.99$ to 1.07$]$ \\
\hline CCI & $1.84[1.77$ to 1.92$]$ \\
\hline Antiplatelet & $1.40[1.23$ to 1.63$]$ \\
\hline Anticoagulant & $3.57[2.22$ to 5.61$]$ \\
\hline ACEI/ARB & $1.53[1.42$ to 1.66$]$ \\
\hline Diuretics & $1.40[1.15$ to 1.69$]$ \\
\hline Chemotherapy & 1.55 [1.16 to 2.09$]$ \\
\hline \multicolumn{2}{|l|}{ Type of anesthesia } \\
\hline General anesthesia & $5.42[4.85$ to 6.03$]$ \\
\hline Regional anesthesia & $3.14[2.89$ to 3.44$]$ \\
\hline Sedation & $2.19[2.05$ to 2.34$]$ \\
\hline Unknown & $0.63[0.58$ to 0.66$]$ \\
\hline Ophthalmologic procedure & 1.58 [1.47 to 1.69$]$ \\
\hline Outpatient & 0.37 [0.35 to 0.39$]$ \\
\hline \multicolumn{2}{|l|}{ Institutional factors } \\
\hline Hospital with $\geq 100$ beds & $2.64[2.53$ to 2.75$]$ \\
\hline Teaching hospital & $0.71[0.66$ to 0.77$]$ \\
\hline Number of operations & 1.15 [1.13 to 1.17$]$ \\
\hline \multicolumn{2}{|c|}{$\begin{array}{l}\text { CEI, angiotensin-converting enzyme inhibitor; ARB, angiotensin-recepto } \\
\text { blocker; CCI, Charlson comorbidity index; HPD, highest posterior density } \\
\text { OR; odds ratio. Notes. The posterior mean of each parameter was used as th } \\
\text { model coefficient, and these values were expressed as odds ratios }\left(\exp \left(\beta_{k}\right)=\right. \\
\text { odds ratio). }\end{array}$} \\
\hline
\end{tabular}

more than 9,000 institutions in Japan [24], suggesting that our results may be highly generalizable. As we adopted a Bayesian approach with the institutions set as random effects, our results regarding the dispersion of this parameter can also be extrapolated to real-world settings. Our Bayesian generalized linear mixed model approach indicated that the adjusted odds ratio for institutions with $\geq 100$ beds was $>2.5$ and that the number of beds was the most influential of the institutional factors investigated. The convergence of the MCMC results is slightly challenging because of strong autocorrelation. Thinning, or other such techniques (e.g., extension of the chain) with at least two or three parallel chains per parameter, can improve the accuracy of the analysis [25]. However, these results were consistent with those of an additional univariate model analysis (OR [95\% HPD interval], 4.06 [3.93 to 4.21]), highlighting the robustness of our finding (univariate model analysis for other predictors are included in Appendix 3). Furthermore, a Bayesian association analysis among predictors which was not planned for our current study may be able to improve the impact of our analysis $[26,27]$.

The 95\% CrI for differences between institutions with $<100$ beds and $\geq 100$ beds was very narrow (approximately $1 \%)$. The use of Bayesian approaches for the calculation of the CrI is advantageous in that the true parameter is contained within the interval $[28,29]$. Thus, our findings strongly suggest that institutional factors influence that rate of preoperative blood testing, consistent with the findings of 
TABLE 3: Differences in the rate of preoperative blood testing based on institution size.

\begin{tabular}{|c|c|c|c|}
\hline & \multicolumn{2}{|c|}{ Preoperative blood test } & \multirow{2}{*}{ Total } \\
\hline & - & + & \\
\hline \multicolumn{4}{|l|}{ Number of beds } \\
\hline$<100$ & $22164(53.9)$ & $18993(46.1)$ & 41157 \\
\hline$\geq 100$ & $6266(22.3)$ & $21829(77.7)$ & 28095 \\
\hline Difference (95\% CrI) & & & $\theta$ \\
\hline $0.315[0.309$ to 0.322$]$ & & & 1.00 \\
\hline
\end{tabular}

CrI, credible interval; $\theta$, Bayesian index.

previous Canadian studies $[9,17]$. Institutions with a large number of beds frequently performed preoperative blood tests, which may have been due to the following reasons: (1) institutions with a large number of beds have enough resources to perform routine laboratory tests regardless of patient status and (2) such testing may be routine at larger institutions. Our findings suggest that clear guidelines for preoperative blood testing are required to avoid unnecessary laboratory tests.

The present study possesses several limitations of note. The main limitation of this study was the use of a database with limited information, as the database did not include information regarding symptoms or physical examination results. In addition, the number of operations could be only analyzed as quantile-categories. Therefore, we were unable to strictly evaluate the suitability of preoperative blood tests for each patient in the present study. In addition, the database contained information from a limited population of participants. Because the database contained only insurance claim-based data accumulated from medium-to-large scale companies in Japan, it only included data for employees under the age of 75 and their families. Therefore, we were unable to examine the influence of patient age on preoperative blood testing. Indeed, previous studies have reported that advanced age is a risk factor for perioperative events and complications, even in low-risk surgeries [30]. Additional investigations involving older adults are thus required to more fully elucidate the influence of patient-based and/or institutional-based factors on the rate of preoperative blood testing.

\section{Conclusion}

In conclusion, our findings indicate that preoperative blood testing prior to low-risk surgery is influenced by institutional factors, such as institution size, suggesting that Bayesian approaches can be used to develop guidelines aimed at reducing excessive preoperative testing. Future studies should investigate the influence of additional patient characteristics (e.g., age) in a more varied population in order to establish the most appropriate guidelines.

\section{Conflicts of Interest}

Koji Kawakami received honoraria from Astellas, Taisho Pharmaceutical, AbbVie, Eisai, Mitsubishi Tanabe Pharma, Takeda Pharmaceutical Company Limited, Sanofi K. K., and consulting fees from Olympus, Kyowa Hakko Kirin, Kaken Pharmaceutical, and Otsuka Pharmaceuticals. There are no patents, products under development, or marketed products relevant to these companies to declare. These companies had no role in the study design, collection, analysis, or interpretation of the data; writing of the manuscript; or the decision to submit the paper for publication. The other authors declare no conflicts of interest.

\section{Authors' Contributions}

Kazuki Ide, Hiroshi Yonekura, and Yohei Kawasaki had full access to all data in the study and take responsibility for the integrity of the data and the accuracy of the data analysis. Study concept and design were performed by Kazuki Ide and Yohei Kawasaki. Acquisition of data was performed by Hiroshi Yonekura and Koji Kawakami. Statistical analysis and interpretation of data were performed by Kazuki Ide, Yohei Kawasaki, and Hiroshi Yonekura. Drafting and revising of the manuscript were performed by Kazuki Ide and Yohei Kawasaki. All authors have read and approved the final manuscript.

\section{Acknowledgments}

The authors would like to acknowledge the staff members at Japan Medical Data Center Co., Ltd., Tokyo, Japan, for assistance with data preparation.

\section{Supplementary Materials}

Appendix 1. MCMC trace and MCMC autocorrelation function plots. Appendix 2. SAS code for analysis. Appendix 3. Univariate analysis for patient and institutional factors. (Supplementary Materials)

\section{References}

[1] C. K. Cassel and J. A. Guest, "Choosing wisely: helping physicians and patients make smart decisions about their care," The Journal of the American Medical Association, vol. 307, no. 17, pp. 1801-1802, 2012.

[2] I. L. Vegting, M. van Beneden, M. H. Kramer, A. Thijs, P. J. Kostense, and P. W. Nanayakkara, "How to save costs by reducing unnecessary testing: Lean thinking in clinical practice," European Journal of Internal Medicine, vol. 23, no. 1, pp. 70-75, 2012. 
[3] C. L. Chen, G. A. Lin, N. S. Bardach et al., "Preoperative medical testing in medicare patients undergoing cataract surgery," The New England Journal of Medicine, vol. 372, no. 16, pp. 1530-1538, 2015.

[4] M. S. Kale, T. F. Bishop, A. D. Federman, and S. Keyhani, "top 5 lists top \$5 billion," Archives of Internal Medicine, vol. 171, no. 20, pp. 1858-1859, 2011.

[5] O. D. Schein, J. Katz, E. B. Bass et al., "The value of routine preoperative medical testing before cataract surgery," The New England Journal of Medicine, vol. 342, no. 3, pp. 168-175, 2000.

[6] G. M. Cavallini, P. Saccarola, R. D’Amico, A. Gasparin, and L. Campi, "Impact of preoperative testing on ophthalmologic and systemic outcomes in cataract surgery," European Journal of Ophthalmology, vol. 14, no. 5, pp. 369-374, 2004.

[7] ABIM Foundation. Choosing Wisely recommendations, http:// www.choosingwisely.org/wp-content/uploads/2015/01/Choosing-Wisely-Recommendations.pdf, 2017.

[8] L. Vogel, "Choosing Wisely around the world," Canadian Medical Association Journal, vol. 187, no. 11, pp. E341-E342, 2015.

[9] K. R. Kirkham, D. N. Wijeysundera, C. Pendrith et al., "Preoperative testing before low-risk surgical procedures," Canadian Medical Association Journal, vol. 187, no. 11, pp. E349-E358, 2015.

[10] Five Things Physicians and Patients Should Question, http://www .choosingwiselycanada.org/wp-content/uploads/2014/01/ENCollection.pdf, 2017.

[11] L. A. Fleisher, J. A. Beckman, K. A. Brown et al., "American College of Cardiology/American Heart Association Task Force on Practice Guidelines (Writing Committee to Revise the 2002 Guidelines on Perioperative Cardiovascular Evaluation for Noncardiac Surgery); American Society of Echocardiography; American Society of Nuclear Cardiology; Heart Rhythm Society; Society of Cardiovascular Anesthesiologists; Society for Cardiovascular Angiography and Interventions; Society for Vascular Medicine and Biology; Society for Vascular Surgery," Circulation, vol. 116, no. 17, pp. e418-e99, 2007.

[12] National Institute for Health and Care Excellence. Preoperative tests (update), https://www.nice.org.uk/guidance/indevelopment/gid-cgwave0689, 2017.

[13] S. Brownlee, K. Chalkidou, J. Doust et al., "Evidence for overuse of medical services around the world," The Lancet, vol. 390, no. 10090, pp. 156-168, 2017.

[14] Y. Zhao, J. Staudenmayer, B. A. Coull, and M. . Wand, "General design Bayesian generalized linear mixed models," Statistical Science. A Review Journal of the Institute of Mathematical Statistics, vol. 21, no. 1, pp. 35-51, 2006.

[15] Y. Kawasaki and E. Miyaoka, "A Bayesian inference of $P(\pi 1>$ $\pi 2$ ) for two proportions," Journal of Biopharmaceutical Statistics, vol. 22, no. 3, pp. 425-437, 2012.

[16] L. G. Glance, S. J. Lustik, E. L. Hannan et al., "The Surgical Mortality Probability Model," Annals of Surgery, vol. 255, no. 4, pp. 696-702, 2012.

[17] K. R. Kirkham, D. N. Wijeysundera, C. Pendrith et al., "Preoperative Laboratory Investigations," Anesthesiology, vol. 124, no. 4, pp. 804-814, 2016.

[18] Y. Fong, H. Rue, and J. Wakefield, "Bayesian inference for generalized linear mixed models," Biostatistics, vol. 11, no. 3, pp. 397-412, 2010.

[19] E. Lesaffre and A. B. Lawson, Bayesian biostatistics, John Wiley and Sons, Hoboken, NJ, USA, 2012.

[20] Japan Medical Association Research Institute. Working paper 2011, http://www.jmari.med.or.jp/download/WP238.pdf, 2017.
[21] World Medical Association, "World Medical Association declaration of Helsinki ethical principles for medical research involving human subjects," Journal of the American Medical Association, vol. 310, no. 20, pp. 2191-2194, 2013.

[22] Ministry of Health, Labour and Welfare., Ethical Guidelines for Medical and Health Research Involving Human, http://www .mhlw.go.jp/file/06-Seisakujouhou-10600000-Daijinkanboukouseikagakuka/0000153339.pdf.

[23] E. von Elm, D. G. Altman, M. Egger, S. J. Pocock, P. C. Gøtzsche, and J. P. Vandenbroucke, "The strengthening the reporting of observational studies in epidemiology (STROBE) statement: guidelines for reporting observational studies," International Journal of Surgery, vol. 12, no. 12, pp. 1495-1499, 2014.

[24] Japan Medical Data Center. “JMDC Claims Database,” https:// www.jmdc.co.jp/en/about/database.html, 2017.

[25] S. M. Lynch, Introduction to Applied Bayesian Statistics and Estimation for Social Scientists, Springer New York, New York, NY, USA, 2007.

[26] J. Ghosh and A. E. Ghattas, "Bayesian variable selection under collinearity," The American Statistician, vol. 69, no. 3, pp. 165$173,2015$.

[27] J. M. Stanton, Reasoning with Data. An Introduction to Traditional and Bayesian Statistics Using R, 2017.

[28] J. J. Lee and C. T. Chu, "Bayesian clinical trials in action," Statistics in Medicine, vol. 31, no. 25, pp. 2955-2972, 2012.

[29] M. J. Bayarri and J. O. Berger, "The interplay of Bayesian and frequentist analysis," Statistical Science. A Review Journal of the Institute of Mathematical Statistics, vol. 19, no. 1, pp. 58-80, 2004.

[30] J. Benarroch-Gampel, K. M. Sheffield, C. B. Duncan et al., "Preoperative Laboratory Testing in Patients Undergoing Elective, Low-Risk Ambulatory Surgery," Annals of Surgery, vol. 256, no. 3, pp. 518-528, 2012. 


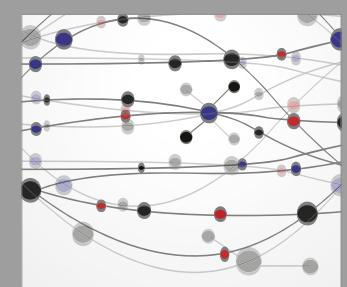

The Scientific World Journal
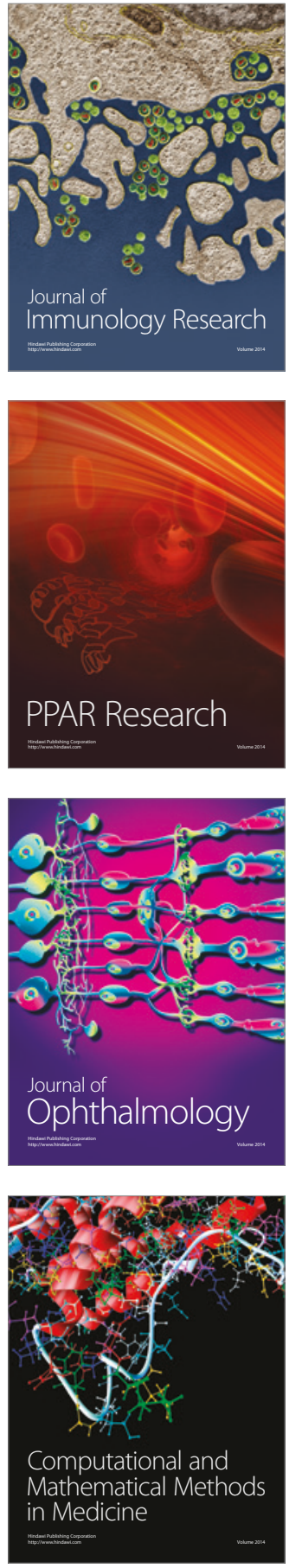

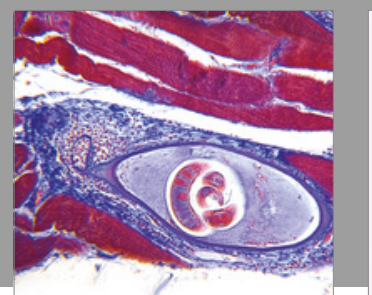

Gastroenterology Research and Practice
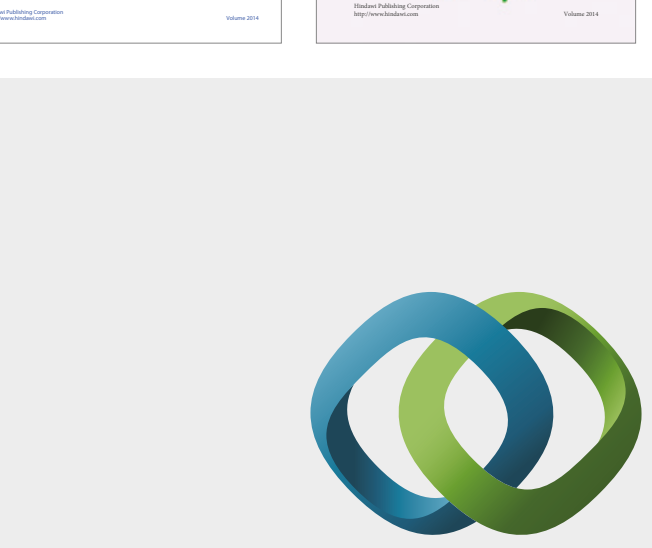

\section{Hindawi}

Submit your manuscripts at

https://www.hindawi.com
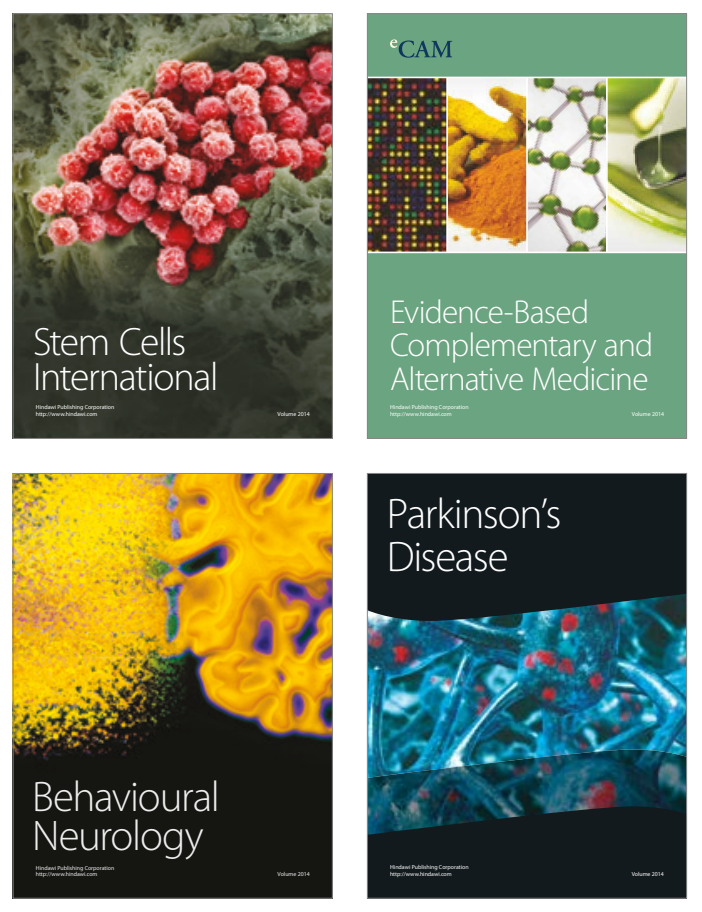
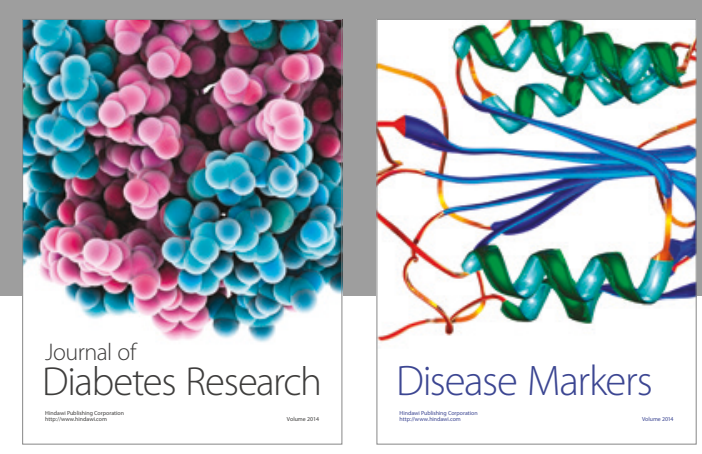

Disease Markers
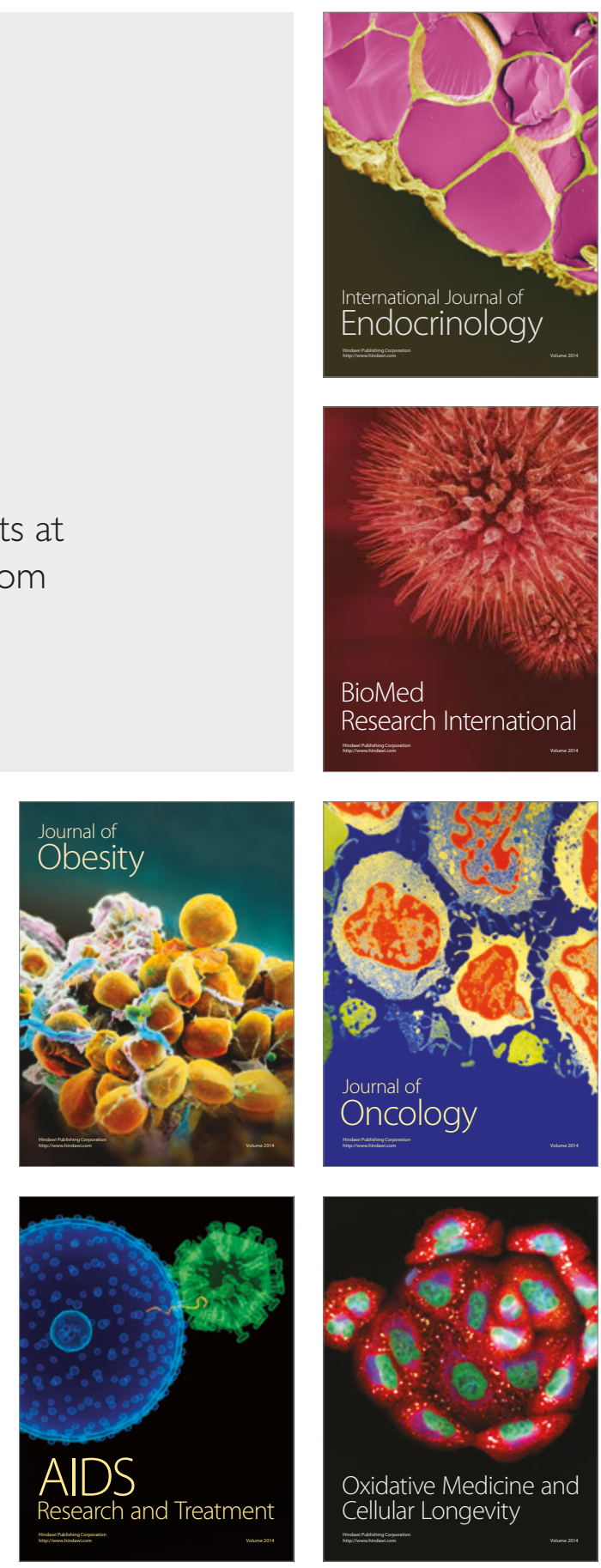